\title{
Components of Lens Power That Regulate Surface Principal Powers and Relative Meridians Independently
}

\author{
H. Abelman ${ }^{1}$ and S. Abelman ${ }^{2}$ \\ ${ }^{1}$ School of Electrical and Information Engineering, University of the Witwatersrand, Johannesburg, \\ Private Bag 3, Wits 2050, South Africa \\ ${ }^{2}$ School of Computer Science and Applied Mathematics, University of the Witwatersrand, \\ Johannesburg, Private Bag 3, Wits 2050, South Africa \\ Correspondence should be addressed to H. Abelman; herven.abelman@wits.ac.za
}

Received 3 February 2016; Revised 17 April 2016; Accepted 29 May 2016

Academic Editor: Nicusor Iftimia

Copyright (c) $2016 \mathrm{H}$. Abelman and S. Abelman. This is an open access article distributed under the Creative Commons Attribution License, which permits unrestricted use, distribution, and reproduction in any medium, provided the original work is properly cited.

\begin{abstract}
Paraxial light rays incident in air on alternate refracting surfaces of a thick lens can yield complementary powers. This paper aims to test when these powers are invariant as surface refractive powers interchange in the expression. We solve for relevant surface powers. Potential anticommutators yield the nature of surface principal refractions along obliquely crossing perpendicular meridians; commutators yield meridians that align with those on the next surface. An invariant power component orients relative meridians or the nature of the matrix power on each noncylindrical surface demands that the other component varies. Another component of lens power aligns relative meridian positions for distinct principal powers. Interchanging surface power matrices affects this component. A symmetric lens power results if perpendicular principal meridians are associated with meridians on an opposite rotationally symmetric surface. For thin lenses, meridian alignment may be waived. An astigmatic contact lens can be specified by symmetric power despite having separated surfaces.
\end{abstract}

\section{Introduction}

Curved lens surface elements close to an optical axis relative to the radii of the surfaces receive paraxial rays. Such elements and the pole of the surface are in sensibly equivalent planes transverse to the axis. Each refracting surface of a lens approximates a plane transverse to the axis, and the thickness of a lens is the separation of these planes, constant everywhere for rays, and equal to the axial thickness $t$ of the lens. We assume a uniform refractive index $n$ in air. Light rays encounter elements on separated surfaces say 1 and 2, with rotational symmetry for which meridians chosen for their distinct powers do not exist. Such a lens has the scalar power given by

$$
G=F_{1}+F_{2}-\frac{t F_{1} F_{2}}{n}
$$

known as Gullstrand's equation [1]. Rays from air may enter on either face without affecting this lens power. Meridians (none is preferred) on one surface necessarily align with those on the other surface. Power $F_{1}$ of surface 1 is conjugate to power $F_{2}$ of surface 2 and powers are called conjugate variables since when these scalars interchange, the expression for $G$ is left invariant by the transposition. Physical conjugates are ubiquitous in techniques and principles of optics and eye care [2].

In the next sections, preferred meridians of lesser symmetry introduced to each lens surface moderate rotational symmetry. Both refracting surfaces may have principal powers along meridians that may be perpendicular or oblique, aligning or crossing obliquely. Let light from air be refracted by a "back" surface of a stationary nonflipped lens. Then in expressions like (1) transposed powers denote that rays meet surfaces in a new order. Matrix surface powers that yield a symmetric or asymmetric invariant power $G$ are found. Previous work considered systems with asymmetric powers [3]. Independent coefficients of lens thickness measuring the effect of transposition may be commutators or anticommutators of lens surface powers and are made explicit in the expression for lens power. We also show that 
principal meridians on the respective lens surfaces are aligned as real surface powers commute [4]. Further, if surface powers anticommute, possible principal powers on lens surfaces are equal-and-opposite powers that cross obliquely at $45^{\circ}$. Matrices in commutators have coincident eigenvectors and coincident eigenvectors are those of commuting matrices. Surface principal powers along oblique meridians [5] or perpendicular meridians that are not aligned are reasons for lenses to have antisymmetric dioptric power matrices. Symmetry of matrices serves as a frame of reference and leads to knowledge about the problem that can be identified with the eigenvectors often measured by instruments in the consulting area.

\section{Method}

Let surface 1 of a lens have matrix power $\mathbf{F}_{1}$ and let the opposite surface 2 have power $\mathbf{F}_{2}$. Suppose principal meridians on lens surfaces cross obliquely. From surfaces 1 to 2, a power matrix of the lens may be [6]

$$
\mathbf{G}=\mathbf{F}_{1}+\mathbf{F}_{2}-\frac{t \mathbf{F}_{2} \mathbf{F}_{1}}{n}
$$

called the Gullstrand equation, generalized in that it follows from rays traced through toric surfaces with matrix powers $\mathbf{F}_{1}$ and $\mathbf{F}_{2}$ [4]. A lens is stationary and as surface powers in expressions are interchanged, this transposition represents light first incident on a "back" refracting surface. If the first two components of an asymmetric power $\mathbf{G}$ are symmetric, this work shows that the component $t \mathbf{F}_{2} \mathbf{F}_{1} / n$ may be asymmetric with the principal powers of the lens generally along oblique meridians. Explicit answers are available for when the principal meridians for separate lens surfaces are aligned and the nature then of the power of the lens. Does anticommutation of surface powers (see (4)) confirm this? How are powers $\mathbf{F}_{1}$ and $\mathbf{F}_{2}$ related for them to interchange in components of (2) such that $\mathbf{G}$ is invariant or not (the lens has not been flipped)?

If the thickness of the lens is neglected, simultaneous equations $t \mathbf{F}_{2} \mathbf{F}_{1} / n=\mathbf{O}$ and

$$
\mathbf{F}_{1}+\mathbf{F}_{2}=\mathbf{G}_{0}
$$

are satisfied in (2). The square matrices $\mathbf{F}_{1}$ and $\mathbf{F}_{2}$ with the same dimensions are conjugate surface powers that are added in any order (associative) leaving the expression for power $\mathbf{G}_{0}$ of the thin lens invariant. A surface with oblique principal meridians contributes an antisymmetric component in (3) so that the matrix $\mathbf{G}_{0}$ is asymmetric [5] and can be expressed as four components: sphere, cylinder, axis, and asymmetry [7].

In lens power equation (2) the coefficient of $t / n$ benefits from the decomposition of the lens surface power products into bracketed terms with noteworthy distinct clinical meanings seen as likely commutators and anticommutators in the identity

$$
2 \mathbf{F}_{2} \mathbf{F}_{1}=\left(\mathbf{F}_{2} \mathbf{F}_{1}-\mathbf{F}_{1} \mathbf{F}_{2}\right)+\left(\mathbf{F}_{2} \mathbf{F}_{1}+\mathbf{F}_{1} \mathbf{F}_{2}\right)
$$

that may each contribute to the symmetry of lens power. If the lens thickness is neglected, these independent meaningful potential symmetry components in (2) and (4) play no role in the lens power as in (3).

We select the left bracket in (4) to write another independent component of lens power $\mathbf{G}$ as

$$
\mathbf{G}_{B}=\frac{t\left(\mathbf{F}_{1} \mathbf{F}_{2}-\mathbf{F}_{2} \mathbf{F}_{1}\right)}{2 n} .
$$

The power in (5) becomes $-\mathbf{G}_{B}$ when $\mathbf{F}_{2}$ and $\mathbf{F}_{1}$ interchange so that surface power $\mathbf{F}_{2}$ is not conjugate to $\mathbf{F}_{1}$. This is the only power component of the lens with this property. The reader can confirm that $\mathbf{G}_{B}$ is the nonzero antisymmetric component of the power $\mathbf{G}$ of a lens whose surfaces are toric but only if surface meridians are obliquely crossed. In (5) commuting matrices $\left(\mathbf{F}_{1} \mathbf{F}_{2}=\mathbf{F}_{2} \mathbf{F}_{1}\right)$ imply alignment of principal meridians of surfaces. We return to this point after (8).

The remaining simultaneous contrasting matrix component of $\mathbf{G}$ follows from the term in the right bracket in (4):

$$
\mathbf{G}_{A}=\mathbf{F}_{1}+\mathbf{F}_{2}-\frac{t\left(\mathbf{F}_{1} \mathbf{F}_{2}+\mathbf{F}_{2} \mathbf{F}_{1}\right)}{2 n},
$$

where $\mathbf{G}_{0}$ in (3) is included in $\mathbf{G}_{A}$ in which surface power $F_{2}$ is seen to be a conjugate of $\mathbf{F}_{1}$ since $\mathbf{G}_{A}$ is invariant when interchanging powers in (6) and it is immaterial whether rays first encounter the lens on surface 1 or 2 . In addition $\mathbf{G}_{A}$ is the symmetric matrix component of the power of a thick lens whose surfaces are toric and $\mathbf{G}_{A}$ is closest to antisymmetric $\mathbf{G}$ in that the Frobenius norm $\left\|\mathbf{G}-\mathbf{G}_{A}\right\|$ is a minimum [8]. With reference to (2), (5), and (6) two components of the matrix power of the lens are

$$
\mathbf{G}=\mathbf{G}_{A}+\mathbf{G}_{B} .
$$

As $\mathbf{F}_{1}$ and $\mathbf{F}_{2}$ trade places in (7), $\mathbf{G}_{B}$ changes sign and power of the lens becomes [6]

$$
\mathbf{G}=\mathbf{G}_{A}-\mathbf{G}_{B} .
$$

Powers of surfaces with preferred meridians have been interchanged for matrices $\mathbf{G}$ from (7) to (8). For lens powers $\mathbf{G}$ not to change, surface matrix power $\mathbf{F}_{1}$ becomes the conjugate matrix power of $F_{2}$ and invariant $G_{A}$ of (6) is the power for a thick lens. For this $\mathbf{G}_{B}=\mathbf{O}$ or

$$
\mathbf{F}_{2} \mathbf{F}_{1}-\mathbf{F}_{1} \mathbf{F}_{2}=\mathbf{O}
$$

or the powers of the refracting surfaces commute. Equation (9) can be shown to be four dependent scalar equations. The solution for the surface powers $\mathbf{F}_{1}$ and $\mathbf{F}_{2}$ of a thick lens requires arbitrary real constants $p$ and $q$ and $\mathbf{I}$ the $2 \times 2$ identity matrix. Matrices $\mathbf{F}_{1}$ and $p \mathbf{F}_{1}+q \mathbf{I}$ can be shown to each have distinct eigenvalues. They represent distinct principal powers that can differ on respective lens surfaces. Different matrices $\mathbf{F}_{1}$ and $\mathbf{F}_{2}=p \mathbf{F}_{1}+q \mathbf{I}$ have a common set of eigenvectors if and only if matrices commute as in (9) [9]. Eigenvectors represent aligning principal meridian directions on respective lens surfaces. Surface powers that commute have principal values along meridians that align from surface to surface and conversely. Equation (9) for thick lenses is valid for the following reason [10]. Pre- and postmultiply $\mathbf{F}_{2}$ by $\mathbf{F}_{1}$ :

$$
\begin{aligned}
& \mathbf{F}_{1} \mathbf{F}_{2}=\mathbf{F}_{1} p \mathbf{F}_{1}+\mathbf{F}_{1} q \mathbf{I}, \\
& \mathbf{F}_{2} \mathbf{F}_{1}=p \mathbf{F}_{1} \mathbf{F}_{1}+q \mathbf{I} \mathbf{F}_{1}
\end{aligned}
$$


TABLE 1: Aligned meridians and commuting surface power matrices. The reader can match the symmetry on respective lens surfaces of meridians in the first column with those in the first row. Meridians have been aligned on surfaces where possible. From (9) power matrices commute for aligned meridians since their power matrices have equal eigenvectors: $\mathbf{F}_{2}=p \mathbf{F}_{1}+q \mathbf{I}$. At not-equal signs, the meridians from opposite lens surfaces cannot physically align.

\begin{tabular}{llll}
\hline & Rotational symmetry & Oblique meridians & Toric symmetry \\
\hline Rotational symmetry & $\mathbf{F}_{1} \mathbf{F}_{2}-\mathbf{F}_{2} \mathbf{F}_{1}=\mathbf{O}$ & $\mathbf{F}_{1} \mathbf{F}_{2}-\mathbf{F}_{2} \mathbf{F}_{1}=\mathbf{O}$ & $\mathbf{F}_{1} \mathbf{F}_{2}-\mathbf{F}_{2} \mathbf{F}_{1}=\mathbf{O}$ \\
Oblique meridians & $\mathbf{F}_{1} \mathbf{F}_{2}-\mathbf{F}_{2} \mathbf{F}_{1}=\mathbf{O}$ & $\mathbf{F}_{1} \mathbf{F}_{2}-\mathbf{F}_{2} \mathbf{F}_{1}=\mathbf{O}$ & $\mathbf{F}_{1} \mathbf{F}_{2} \neq \mathbf{F}_{2} \mathbf{F}_{1}$ \\
Toric symmetry & $\mathbf{F}_{1} \mathbf{F}_{2}-\mathbf{F}_{2} \mathbf{F}_{1}=\mathbf{O}$ & $\mathbf{F}_{1} \mathbf{F}_{2} \neq \mathbf{F}_{2} \mathbf{F}_{1}$ & $\mathbf{F}_{1} \mathbf{F}_{2}-\mathbf{F}_{2} \mathbf{F}_{1}=\mathbf{O}$ \\
\hline
\end{tabular}

so that these matrices commute as in (9). Thus surface principal powers along arbitrary aligning meridians of a thick lens satisfy $\mathbf{F}_{2}=p \mathbf{F}_{1}+q \mathbf{I}$ and have a power matrix $\mathbf{G}_{A}$ that remains unchanged. A lensometer measures back and front surface vertex powers and may not be the general detector for lens power $\mathbf{G}_{A}$. Since $\mathbf{F}_{1}$ and $\mathbf{F}_{2}=p \mathbf{F}_{1}+q \mathbf{I}$ have a common set of linearly independent eigenvectors [9], (9) is valid and $\mathbf{F}_{1}$ and $\mathbf{F}_{2}$ commute. Only one of $\mathbf{F}_{1}$ and $\mathbf{F}_{2}$ may represent the power of a surface without preferred meridians (such matrices always commute, (1) and Table 1). For a thick lens, commuting powers $\mathbf{F}_{1}$ and $\mathbf{F}_{2}$ in $\mathbf{G}_{A}$ are conjugates that may be multiplied in any order in their product in (2) and (6).

Another matrix component can have zero power irrespective of lens thickness when

$$
\mathbf{O}=\mathbf{F}_{1} \mathbf{F}_{2}+\mathbf{F}_{2} \mathbf{F}_{1}
$$

or the matrices $\mathbf{F}_{1}$ and $\mathbf{F}_{2}$ anticommute. General surface principal powers for which this is the case are independent of those for which (9) holds. Suppose $\mathbf{F}_{1}$ is invertible. This excludes pure cylinder-shaped surfaces 1 . Then, (11) can be $\mathbf{F}_{2}=\mathbf{F}_{1}^{-1}\left(-\mathbf{F}_{2}\right) \mathbf{F}_{1}$ which makes $\mathbf{F}_{2}$ have the same trace as $-\mathbf{F}_{2}$. But, generally, trace $\left(-\mathbf{F}_{2}\right)=-\operatorname{trace}\left(\mathbf{F}_{2}\right)$. Thus the trace of $\mathbf{F}_{2}$ must be zero so that surface 2 has equal-andopposite principal powers. This procedure is equally valid for $\mathbf{F}_{1}\left(\mathbf{F}_{2}\right.$ is invertible) and each surface is cross-cylinderlike. Anticommuting matrices $\mathbf{F}_{1}$ and $\mathbf{F}_{2}$ in (11) yield equaland-opposite powers on the surfaces. This is not sufficient for (11) to be valid. Further investigation shows that principal meridians must cross at $45^{\circ}$ from one surface to the next. $\mathbf{F}_{1}+\mathbf{F}_{2}$ and $t \mathbf{F}_{1} \mathbf{F}_{2} / n$ are the simultaneous power components in (2) for a lens where neither surface is a pure cylinder. Then the equal-and-opposite powers on separate surfaces can have principal meridians crossing maximally at $45^{\circ}$. Equation (9) cannot be valid with (11) and associated conjugate powers cannot be present on such a thick lens simultaneously. Information on surface powers disappears if the thickness of the lens is neglected as (9) and (11) effectively become valid simultaneously.

Conjecture 1. Energy illuminating the retina should be a significant fraction of the energy illuminating a similar area on the correcting lens. Any oblique astigmatism or asymmetry present in the power of the compensating device makes rays focus as caustic curves. Stray beams illuminate the eyeball. Contrast is thus lowered and less energy concentrates at the fovea. Spectacle or contact lenses manufacturers are trained to machine surfaces that combine and nullify the asymmetry. For efficient use of illumination energy, spherocylindrical lenses have powers that are symmetric, and perpendicular meridians align. The validity of this seems to be supported by common practice motivated in part by this work.

General obliquely crossing principal meridians on surfaces are progressively aligned in what follows, to finally be rectangular. Corresponding lens powers are seen to have the nature that the theory predicts.

Surfaces 1 and 2 of a lens have a common vertical principal meridian $\beta$ whose powers $F_{\beta 1}$ and $F_{\beta 2}$ are different and nonzero in Figure 1. Near-horizontal principal meridians of plano power on the first surface are not aligned with those on the second surface. Angles are $\pi-\alpha_{1}$ on the left and $\pi-\alpha_{2}$ on the right and $\alpha_{1}$ and $\alpha_{2}$ in radians are small. Eigenvector

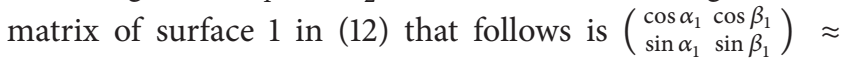
$\left(\begin{array}{cc}-1 & 0 \\ \alpha_{1} & 1\end{array}\right)$ and for surface 2 we have $\left(\begin{array}{cc}\cos \alpha_{2} & \cos \beta_{2} \\ \sin \alpha_{2} & \sin \beta_{2}\end{array}\right) \approx\left(\begin{array}{cc}-1 & 0 \\ \alpha_{2} & 1\end{array}\right)$ with corresponding singular eigenvalue matrices $\left(\begin{array}{cc}0 & 0 \\ 0 & F_{\beta 1}\end{array}\right)$ and $\left(\begin{array}{cc}0 & 0 \\ 0 & F_{\beta 2}\end{array}\right)$. Surface power matrices $\mathbf{F}_{1}$ and $\mathbf{F}_{2}$ are [5]

$$
\begin{aligned}
& \mathbf{F}_{1}=\left(\begin{array}{cc}
-1 & 0 \\
\alpha_{1} & 1
\end{array}\right)\left(\begin{array}{cc}
0 & 0 \\
0 & F_{\beta 1}
\end{array}\right)\left(\begin{array}{cc}
-1 & 0 \\
\alpha_{1} & 1
\end{array}\right), \\
& \mathbf{F}_{2}=\left(\begin{array}{cc}
-1 & 0 \\
\alpha_{2} & 1
\end{array}\right)\left(\begin{array}{cc}
0 & 0 \\
0 & F_{\beta 2}
\end{array}\right)\left(\begin{array}{cc}
-1 & 0 \\
\alpha_{2} & 1
\end{array}\right) .
\end{aligned}
$$

Slightly different principal meridians and powers on the surfaces in Figure 1 are represented in the distinct modal and spectral matrices in (12) that yield asymmetric powers $\mathbf{F}_{1}$ and $\mathbf{F}_{2}$.

Meridians of plano power on both refracting surfaces in Figure 1 are not perpendicular to meridian $\beta$ so that matrices $\mathbf{F}_{1}+\mathbf{F}_{2}$ and $\mathbf{F}_{1} \mathbf{F}_{2}$ are antisymmetric and $\mathbf{G}$ in (2) is an antisymmetric power matrix. Thus surface powers $\mathbf{F}_{1}$ and $\mathbf{F}_{2}$ in the power $\mathbf{G}$ of the lens that transmits light from surface to surface are not conjugates. For $\mathbf{F}_{1}$ and $\mathbf{F}_{2}$ to commute $\left(\mathbf{G}_{B}=\right.$ $\mathbf{O}$ in (5)), surfaces need to have aligning meridians (equal eigenvectors) and for this the matrix products identified by the square brackets in

$$
\begin{aligned}
& \mathbf{F}_{1} \mathbf{F}_{2} \\
& =\left(\begin{array}{ll}
-1 & 0 \\
\alpha_{1} & 1
\end{array}\right)\left(\begin{array}{cc}
0 & 0 \\
0 & F_{\beta 1}
\end{array}\right)\left[\begin{array}{ll}
-1 & 0 \\
\alpha_{1} & 1
\end{array}\right]\left[\begin{array}{cc}
-1 & 0 \\
\alpha_{2} & 1
\end{array}\right]\left(\begin{array}{cc}
0 & 0 \\
0 & F_{\beta 2}
\end{array}\right)\left(\begin{array}{cc}
-1 & 0 \\
\alpha_{2} & 1
\end{array}\right), \\
& \mathbf{F}_{2} \mathbf{F}_{1} \\
& =\left(\begin{array}{ll}
-1 & 0 \\
\alpha_{2} & 1
\end{array}\right)\left(\begin{array}{cc}
0 & 0 \\
0 & F_{\beta 2}
\end{array}\right)\left[\begin{array}{cc}
-1 & 0 \\
\alpha_{2} & 1
\end{array}\right]\left[\begin{array}{cc}
-1 & 0 \\
\alpha_{1} & 1
\end{array}\right]\left(\begin{array}{cc}
0 & 0 \\
0 & F_{\beta 1}
\end{array}\right)\left(\begin{array}{cc}
-1 & 0 \\
\alpha_{1} & 1
\end{array}\right)
\end{aligned}
$$



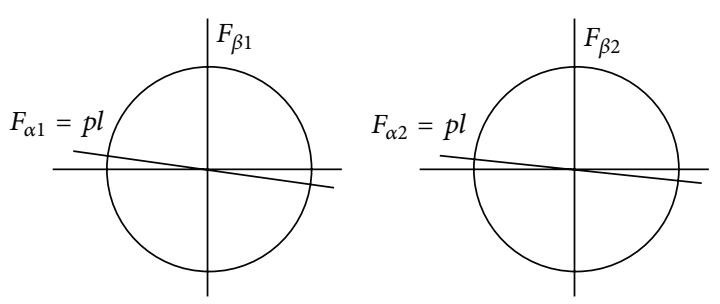

FIGURE 1: Coincident vertical principal meridians whose powers are different are found on the surfaces of a thick lens. Near-horizontal noncoincident meridians have plano power.

equal the identity matrix. This, or equally $\mathbf{F}_{2}=p \mathbf{F}_{1}+q \mathbf{I}$, implies that the meridians of plano power must coincide on the two surfaces, that is, $\alpha_{1}=\alpha_{2}$ while the $\beta$ meridians coincide. These conditions cause antisymmetric $\mathbf{F}_{1}$ to be a conjugate of $\mathbf{F}_{2}$ or $\mathbf{F}_{1}$ and $\mathbf{F}_{2}$ commute as in (9) and $\mathbf{F}_{1} \mathbf{F}_{2}$ is antisymmetric. Equation (2) yields the thick lens of asymmetric power

$$
\mathbf{G}=\left(F_{\beta 1}+F_{\beta 2}-\frac{t F_{\beta 1} F_{\beta 2}}{n}\right)\left(\begin{array}{cc}
0 & 0 \\
\alpha_{1} & 1
\end{array}\right)
$$

from aligned meridians of surfaces with conjugate matrix powers. The meridian of plano power will be horizontal on the respective surfaces when $\alpha_{1}=0$ in Figure 1 . Aligned meridians on the surfaces from the remaining $\mathbf{G}_{A}$ will be perpendicular which yields a thick lens of symmetric power. Equations (7) and (8) show that surface powers that interchange produce a different power for lens power G. Cylindrical surfaces whose principal meridians each are aligned, vertical and horizontal, form the lens. Power $\mathbf{G}$ is determined with respect to a principal plane of the lens. This power may be expressed as sphere, cylinder, and axis but we may not assume that $\mathbf{G}$ can be measured with respect to a back surface of the lens. All modal matrices in (12) have become $\left(\begin{array}{cc}-1 & 0 \\ 0 & 1\end{array}\right)$. The columns (eigenvectors) of this symmetric matrix are along the horizontal and vertical principal meridians of a thick lens that are aligned on the respective refracting surfaces of Figure 1 and whose powers $F_{\beta 1}$ and $F_{\beta 2}$ differ.

\section{Discussion}

In a general lens, the ray path stipulated appropriate independent components of a power matrix. Interchanging surface powers in an expression affected some components. A demand for invariant lens power made surface powers conjugate. The resultant matrix commutation was exploited advantageously to justify the shape and power of surfaces of spherocylinder lenses. Mostly the lens was treated as thick.

The symmetries of powers along lens surface meridians in (1) are substituted by principal meridians along surfaces in the generalized Gullstrand equation (2) in which the power of a compensating lens depends on the refracting surface that rays first encounter. The thickness-dependent second-order term with a generally asymmetric power was written as autonomous components using (4) to facilitate the recognition of conjugates. A component of lens power in (6) is invariant when matrix surface powers, now mutually conjugate, change position in the expression. For toric surfaces, in particular, this is the invariant component of lens power. Principal powers along oblique meridians may be those of conjugate surface power components of invariant lens power. A second power component changed sign when matrix surface powers change position in the expression for lens power and controlled how the meridians cross on respective surfaces. For toric surfaces, in particular, this component of asymmetric lens power peters out as principal meridians, represented by eigenvectors of surface matrix powers, align as in (9). Principal powers are distinct on a surface but differ among surfaces. We showed why meridians on pairs of toric refracting surfaces or surfaces with oblique meridians can align or cross obliquely to determine the natures of thick lens powers. All pairs of surfaces, one of which may not have preferred meridians, have distinct matrix powers that commuted in the expression for lens power when surface eigenvectors aligned as illustrated. For thin lenses, meridian alignment essential for a symmetric thick lens power component may be waived. Power component $\mathbf{G}_{A}$ that is independent of the order of surface powers in its expression provides a norm or reference as paraxial rays do in ray tracing. Component $\mathbf{G}_{B}$ measures the full deviation of power from containing conjugate variables and symmetry and contributes zero to lens power for an appropriate selection of surface variables. Spherocylindrical lenses need to have thickness to maintain adequate mechanical strength and safety. We have shown that lenses with adequate thickness can have toric power allocated to one surface and spherical power to the other or any of the other options as shown in Table 1 where the surface powers commute. Principal planes in rotationally symmetric lenses are scalar conjugates that may have a matrix analogue and we believe that conjugate variables open a possibility for matrices to shape some principal surfaces in spherocylinder lenses in future. The separation of refracting surfaces may not contribute to antisymmetry in the power matrix. Symmetric or asymmetric lens power can thus be invariant when surface powers are switched for this thick lens.

First-order image positions and sizes that paraxial optics predicts in a system provide a convenient reference from which to measure departures from perfection including aberrations. Linear paraxial expressions are simpler than trigonometric equations [11].

Parabolic mirror surfaces that cast telescope images, retinoscopy $[12,13]$, and automated optometers provide experimental answers or are calibrated based on principles present in conjugate variables. A common thread in thermodynamics, statistical physics, Hamiltonian mechanics, and other disciplines is the Legendre transform that contains conjugate variables. Well-known conjugate variables are present in the formats for Heisenberg's uncertainty principle. Standard methods of linear algebra [14] can be applied to fields that include medical imaging, engineering, and ophthalmic literature, where eigenvalues and eigenvectors often represent observable and measureable quantities. 


\section{Competing Interests}

The authors declare that there is no competing interests regarding the publication of this paper.

\section{Acknowledgments}

The University of the Witwatersrand, Johannesburg is thanked for hospitality. S. Abelman gratefully acknowledges support from the University of the Witwatersrand, Johannesburg and the National Research Foundation, Pretoria, South Africa.

\section{References}

[1] L. Wang, A. M. Mahmoud, B. L. Anderson, D. D. Koch, and C. J. Roberts, "Total corneal power estimation: ray tracing method versus Gaussian optics formula," Investigative Ophthalmology and Visual Science, vol. 52, no. 3, pp. 1716-1722, 2011.

[2] A. G. Bennett, "An historical review of optometric principles and techniques," Ophthalmic and Physiological Optics, vol. 6, no. 1, pp. 3-21, 1986.

[3] W. F. Harris and R. D. van Gool, "Thin lenses of asymmetric power," South African Optometrist, vol. 68, no. 2, pp. 52-60, 2009.

[4] W. F. Harris, "Back- and front-vertex powers of astigmatic systems," Optometry and Vision Science, vol. 87, no. 1, pp. 7072, 2010.

[5] H. Abelman and S. Abelman, "Paraxial ocular measurements and entries in spectral and modal matrices: analogy and application," Computational and Mathematical Methods in Medicine, vol. 2014, Article ID 950290, 8 pages, 2014.

[6] R. Blendowske, "Hans-Heinrich Fick. Early contributions to the theory of astigmatic systems," South African Optometrist, vol. 62, no. 3, pp. 105-110, 2003.

[7] W. F. Harris, "Keating's asymmetric dioptric power matrices expressed in terms of sphere, cylinder, axis, and asymmetry," Optometry and Vision Science, vol. 70, no. 8, pp. 666-667, 1993.

[8] H. Abelman and S. Abelman, "Modification of readings along oblique principal meridians to fit regular corneal surfaces," Journal of Modern Optics, vol. 62, no. 14, pp. 1187-1192, 2015.

[9] K. F. Riley, M. P. Hobson, and S. J. Bence, Mathematical Methods for Physics and Engineering, Cambridge University Press, Cambridge, UK, 2006.

[10] C. J. Eliezer, "A note on group commutators of $2 \times 2$ matrices," American Mathematical Monthly, vol. 75, no. 10, pp. 1090-1091, 1968.

[11] W. J. Smith, Modern Optical Engineering, McGraw-Hill, New York, NY, USA, 2000.

[12] M. Rosenfield, N. Logan, and K. H. Edwards, Optometry: Science, Techniques and Clinical Management, ButterworthHeinemann, Edinburgh, UK, 2009.

[13] M. P. Keating, Geometric, Physical, and Visual Optics, Butterworth-Heinemann, Boston, Mass, USA, 1988.

[14] H. Anton and C. Rorres, Elementary Linear Algebra: Applications Version, JohnWiley \& Sons, Philadelphia, Pa, USA, 2014. 

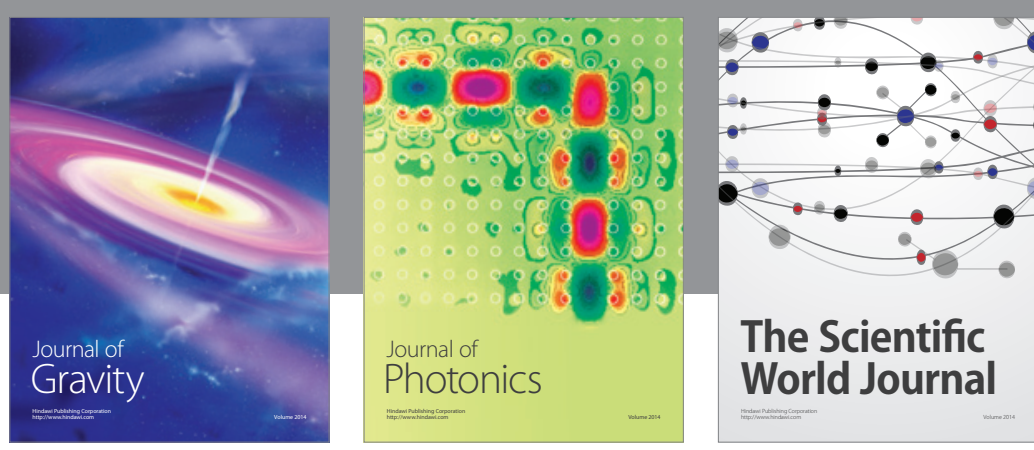

The Scientific World Journal
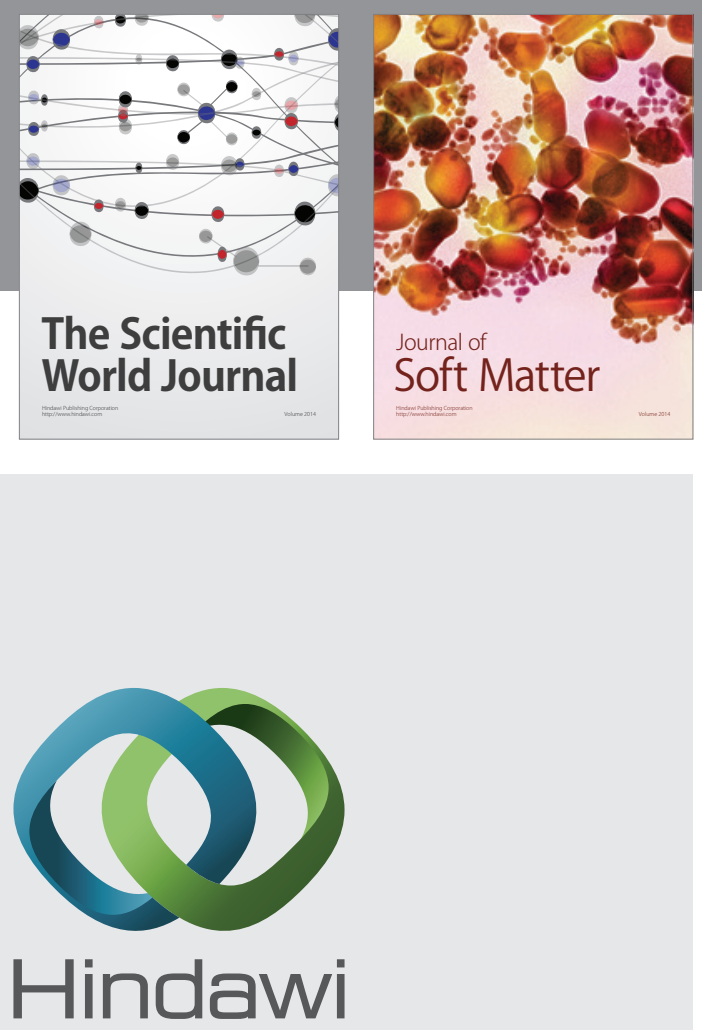

Submit your manuscripts at

http://www.hindawi.com

nternational Journal of

Statistical Mechanics
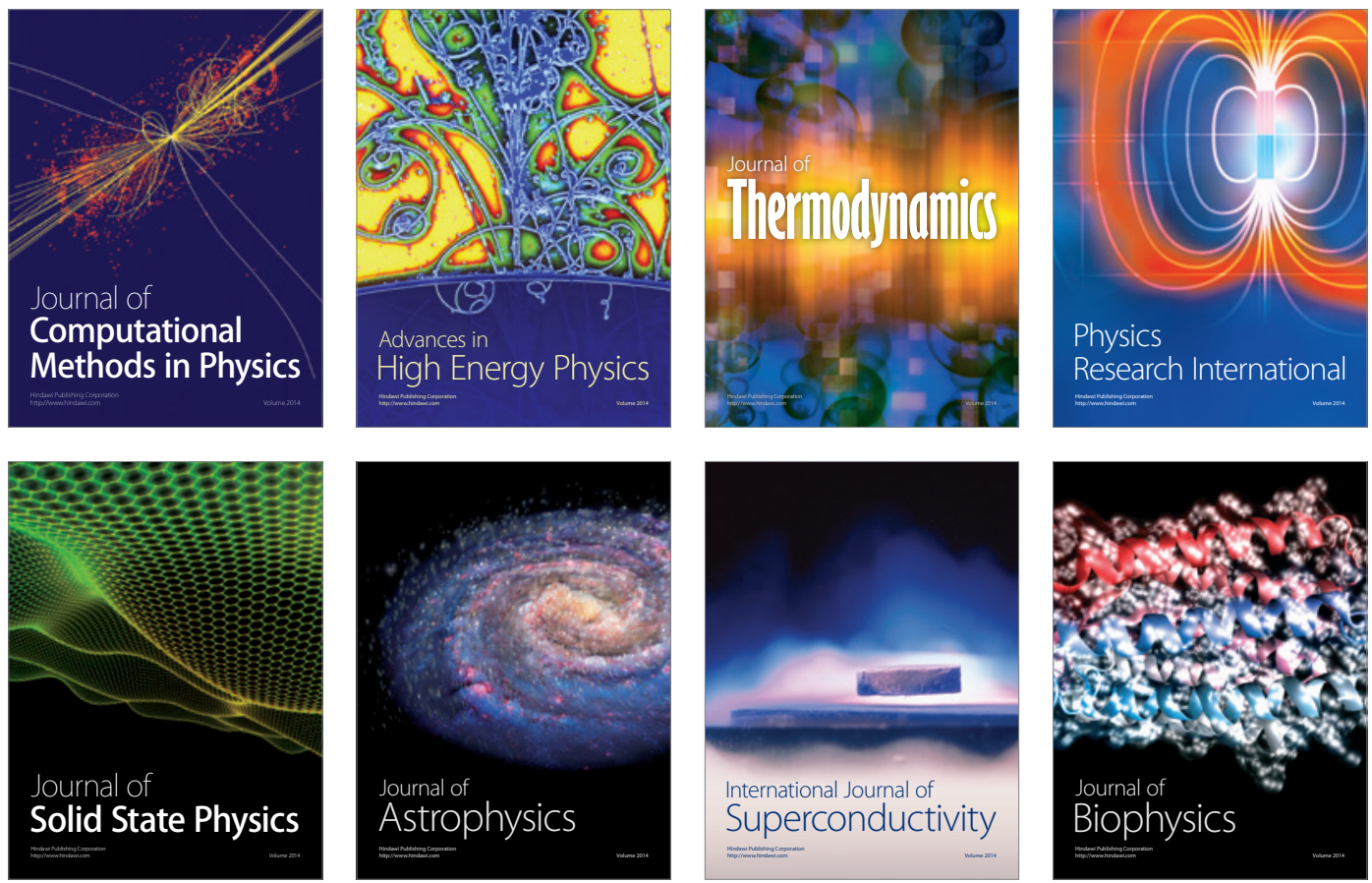
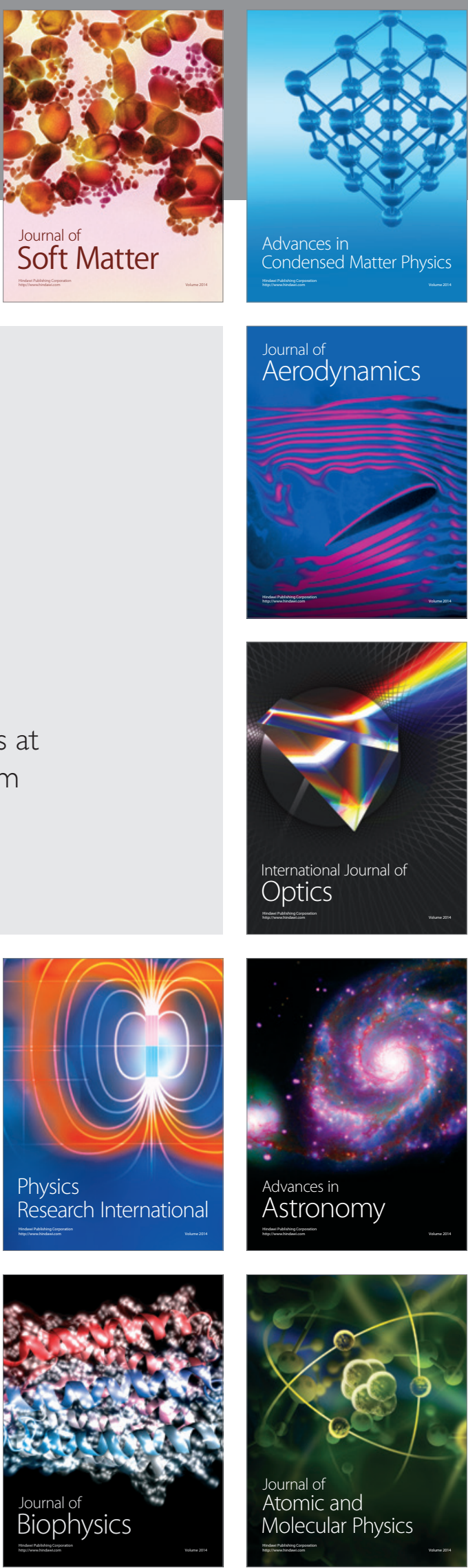